\title{
Benefits of costing in the clinical laboratory*
}

\author{
PMG BROUGHTON, FP WOODFORD \\ From the Wolfson Research Laboratories, Queen Elizabeth Medical Centre, Birmingham and the Department \\ of Health and Social Security, Hannibal House, Elephant and Castle, London
}

SUMMARY This paper urges the benefits of applying more widely a method for pathology laboratory costing originally devised for a clinical chemistry department, and illustrates these with examples drawn from costing studies in three clinical laboratories.

Heads of pathology departments, laboratory managers, administrators and clinicians require different kinds of costing information, each of which can be obtained by the costing procedure outlined. The method also yields valuable and sometimes surprising insights into the workings of a pathology service. Cost comparisons between different laboratories can now become more informative.

Flaws in the concept of the "cost per test" are discussed and the value of this concept is questioned; for most purposes the cost per request has greater application.

In 1981 Broughton and Hogan published a method for costing a clinical chemistry department ${ }^{1}$ which is simpler, more logical and more comprehensible than previously published methods. ${ }^{2-4} \mathrm{~A}$ novel feature of these authors' approach was to separate costs into those involved in providing the basic facility-that is, an appropriately staffed and equipped laboratory competent to fulfil the requests made to it-and the actual direct costs of performing individual test procedures, and to apply these figures separately to answer different types of management question.

So far, there has been no stampede to apply this method in other clinical laboratories, despite the current financial restrictions and the concern about. the cost of "high technology" diagnostic tests. ${ }^{5}$ Reluctance to embark on costing studies stems from a mistaken belief that the amount of effort necessary for reliable costing is incommensurate with the possible benefits.

This paper outlines the principles of the Broughton and Hogan method and explains which elements of the procedure can be used to answer costing questions asked by pathologists, administrators or clinicians. The usefulness of the method is illustrated by

\footnotetext{
*Based on research commissioned by the Department of Health and Social Security at the Wolfson Research Laboratories. The views expressed should not be taken as official Departmental policy on laboratory costing.

Accepted for publication 5 April 1983

(C) Crown copyright 1983.
}

its application to three clinical chemistry laboratories, but there is every reason to believe that it can be applied without change in other pathologe disciplines.

\section{Principles of the costing method of Broughton and Hogan ${ }^{1}$}

Throughout pathology the basic unit of work is the request, representing a clinical question, which may require one or more tests or test groups to be performed. Before devising any costing procedure it is most important to define exactly what is meant by "cost per request" and "cost per test" and why each value is needed. Otherwise, management and planning will be bedevilled by uncertainty and confusion.

A basic requirement for a satisfactory laboratory costing method is the attribution of all items of expenditure to one or other aspect of the service provided. This allows a balance sheet in which the sum of the cost of all individual items equals the known expenditure. Isolated costing of one area of work or of single tests is usually unsatisfactory because the results cannot be cross-checked against the total expenditure.

In order to apportion expenditure between different types of work, direct costs must be identifiedthat is, those costs which are necessarily and exclusively incurred in performing a specified test at a particular time..$^{13}$ These must be distinguished from all other costs, which are termed indirect. A fundamental problem in accounting procedures is the 
allocation of indirect costs. Broughton and Hogan's solution to this problem was not to allocate them to individual tests but to combine them, divide by the total number of requests, and call the resultant quotient a "handling charge per request." This represents the basic cost of having a properly staffed, equipped and quality-controlled laboratory available to meet the demands placed upon it. The total cost, in a given laboratory, of fulfilling a request for a particular investigation is then the sum of this basic handling charge plus the direct costs of carrying out the test $(s)$ to which it gives rise. The advantages of separately calculating the "indirect cost per request" and the "direct cost for each type of test" are real and important.

The direct cost of any given test varies, even within a single laboratory, depending on the total number of such tests performed, the frequency with which they are performed (individually or in batches), and whether they are done in or out of normal working hours. ${ }^{1}$ Nevertheless, the average direct cost for each type of test should be similar in all laboratories, and if a major discrepancy is discovered it will be worth seeking the reasons in the hope that savings can be made-for example, by changing the analytical methods or source of reagents.

The "indirect cost per request" must be expected to differ between laboratories since the indirect costs are largely made up of the salaries of senior and supervisory staff. The indirect cost per request is therefore higher in laboratories which offer a wider range of tests and which have more extensive training, research and consultative functions. It would be difficult to identify laboratories which are directly comparable in all these respects, and this is why the indirect costs should not be muddled in with direct costs in a vain attempt to arrive at a total "cost per test" for comparison between laboratories. It is because indirect costs cannot be allocated to individual tests in any logically satisfying way that Broughton and Hogan elected to divorce them completely from the "cost per test" with which other costing procedures ${ }^{2-4}$ had sought to associate them.

The practicalities of the Broughton and Hogan method are as follows (for details see reference 1). The direct costs of labour and consumables which can be attributed to particular tests or test groups are totalled. This is subtracted from the total annual revenue expenditure of the laboratory, to give the indirect costs of labour and consumables (Tables 1 and 2). No attempt is made to measure the separate items of indirect cost: the total is obtained by subtraction. For calculation of the capital costs, laboratory equipment is divided into that dedicated to particular tests (for calculation of direct costs)

\section{Table 1 Examples of indirect consumables}

General laboratory consumables (glassware, disposables, etc)

Maintenance of indirect equipment (computer, freezers, centrifuges, autoclaves, etc)

Heating and lighting

Rates and taxes

Quality control materials (for multiple tests)

Transport

Telephone and postage

Printing, stationery and other paper

Journals and books

Job advertising

Table 2 Examples of indirect labour

Specimen collection and separation

Lahoratory housekeeping and quality control

Safety arrangements and monitoring

Checking reports

Record keeping and computing

Administration

Training, conferences

Teaching

Research and method development

Committees and consultations

Secretarial and clerical work

Portering and cleaning

Coffee breaks and personal time

Holidays (about $10 \%$ of all staff time)

Sickness

and general purpose equipment (to calculate indirect costs). In each case the current replacement cost is divided by the expected equipment lifespan in years to give the notional capital cost per annum.

In the pathology service of the UK National Health Service, indirect labour costs constitute a very large proportion not only of the labour costs $(80-85 \%$, calculable from Table 3$)$, but of the total

Table 3 Annual revenue expenditure and capital costs of clinical chemistry laboratories $A(1980 / 81)$ and $B$ $(1979 / 80)$ at $1981 / 82$ prices*

\begin{tabular}{|c|c|c|}
\hline & $\begin{array}{l}A \\
(43216 \text { requests }) \\
(£)\end{array}$ & $\begin{array}{l}B \\
\text { (49447 requests) } \\
\text { (f) }\end{array}$ \\
\hline $\begin{array}{l}\text { Direct consumables } \\
\text { Indirect consumables } \\
\text { Direct labour } \\
\text { Indirect labour }\end{array}$ & $\begin{array}{r}21662 \\
25568 \\
19418 \\
105997 \\
\end{array}$ & $\begin{array}{r}32956 \\
42918 \\
47311 \\
188854 \\
\end{array}$ \\
\hline $\begin{array}{l}\text { Total revenue } \\
\text { expenditure }\end{array}$ & $\underline{172645}$ & 312039 \\
\hline $\begin{array}{l}\text { Direct capital costs } \\
\text { Indirect capital costs } \\
\text { Total indirect costs }\end{array}$ & $\begin{array}{r}19624 \\
3895 \\
135460\end{array}$ & $\begin{array}{r}19008 \\
5701 \\
237473\end{array}$ \\
\hline $\begin{array}{l}\text { Total indirect cost } \\
\text { per request }\end{array}$ & $3 \cdot 13$ & 4.80 \\
\hline
\end{tabular}

*The costing studies described in this paper were made at various times over two years. All figures have been corrected to the equivalents for the financial year $1981 / 82$ by multiplying by 1.384 for measurements made in $1979 / 80$, or 1.081 for measurements made in 1980/81 (Hospital and Community Health Service Revenue Revaluation Factors-Pay and Prices, DHSS 1981).

tThe term "revenue expenditure", etymologically a contradiction in terms, is used in the UK to mean non-capital (ie recurrent) expenditure. 
Table 4 Examples of direct and indirect costs in laboratory $B(1979-80)$ at 1981/82 prices

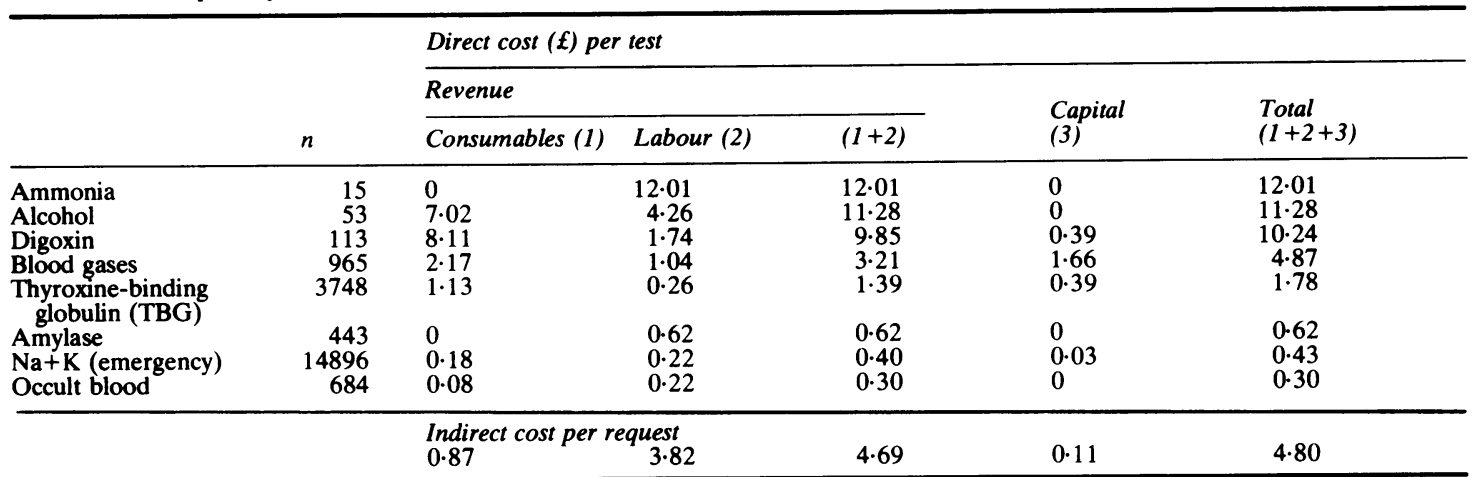

$\mathrm{n}=$ number of tests performed during the year.

cost $(55 \%)$. This reflects not only the need for the laboratory to have sufficient staff to cope effectively with peaks of busy days and urgent work, but also high expenditure on supervision, quality control, training, research and development, as well as the scientific and medical advisory functions of senior laboratory staff. These activities are all characteristic of "good" laboratories, but are open-endedthat is, there is no norm. To curtail them would reduce costs, but would result in a poorer service.

Table 4 shows examples of direct costs of some tests in one laboratory, as well as the indirect cost per request in that laboratory, analysed into consumable, labour and capital components. The cost of each test type may be expressed either as the average direct cost per test performed, which is conventional, or as the total annual direct expenditure on each type of test, which is of greater use to the laboratory manager.

In addition to the average direct cost per test the direct costs of the same tests performed at different times, for example as part of a batch or as an emergency (Table 5), within and out of normal working hours, can be obtained.

Table 5 Direct costs of serum $\mathrm{Na}+K$ assay under different circumstances (1979/80 converted to 1981/82 prices)

\begin{tabular}{llll}
\hline & $n$ & \multicolumn{2}{l}{$\begin{array}{l}\text { Direct revenue cost }(f) \\
\text { per test }\end{array}$} \\
\cline { 2 - 4 } & & Consumables & Labour \\
\hline Profiles (Lab C) & 51,119 & 0.03 & 0.07 \\
Emergencies (Lab B) & 14,896 & 0.18 & $0.22^{*}$ \\
\hline
\end{tabular}

$\mathrm{n}=$ number of test pairs performed during the year.

* If out of normal hours, the labour cost will be much higher'.

\section{Uses of costing data in laboratory management}

Three questions of cost are regularly faced by laboratory managers: what would be the financial consequences of (a) changes in analytical method, reagents or equipment; (b) changes in workload; (c) changes in laboratory organisation and practice?

CHANGES IN ANALYTICAL METHOD

In many cases the financial consequences of change in reagents are clear and simple, but the effects changes in analytical method or equipment are more complex. As an example, consider the effect on revenue expenditure of replacing an existing flame photometer (for the measurement of serum and urine $\mathrm{Na}^{+}$and $\mathrm{K}^{+}$) by a new ion-selective electrode (ISE) analyser, without change in workload. Given that the number of staff employed cannot be changed in the short term, the total labour costs will be unchanged. Only expenditure on direct consumables need therefore be considered. This is made up of fixed costs that are independent of workload, and variable or workload-dependent costs (Table 6).

With a flame photometer, the variable costs are directly related to the running time of the instrument, but for the ISE analyser they depend on the workload, batch size and time between batches. Furthermore, the ISE analyser incurs direct costs even when no specimens are analysed. Consequently the relation between costs and workload is not a simple one. The calculations in Table 6, which are given for illustrative purposes only, are based on the actual workload of an emergency laboratory analysing serum and urine specimens by day and 0 night. They show that use of the ISE analyser to deal $\overparen{D}$ with this workload distribution would increase the $\stackrel{?}{\rightarrow}$ annual expenditure on consumables by $£ 3116$. Expressing the data in this form also allows calcula- 
Table 6 Costs (adjusted to 1981/82 prices) of analysing 15481 sera and 3889 urines per annum for $\mathrm{Na}+\mathrm{K}$ with an ISE analyser and a flame photometer $(\mathrm{Na}+\mathrm{K}$ is considered as a single test)

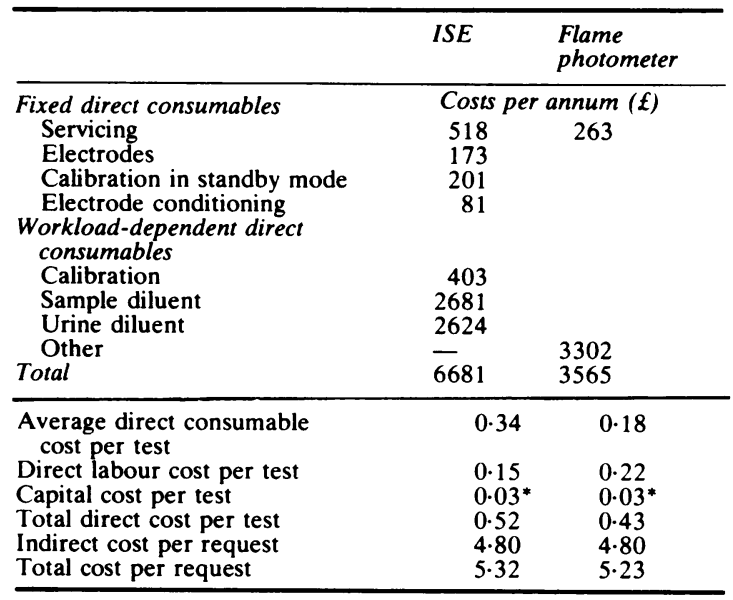

* The prices of the two instruments were very similar.

tions to be made for different workloads.

If such an ISE analyser were installed in a clinical area to do some of the work previously done by the emergency laboratory, labour costs in both locations would be affected, but these would be impossible to predict without detailed timing studies.

The data in the lower part of Table 6 show that the direct cost per test, which appears small for either method, does not so much as hint at the large total annual expenditure on the test. Tests which are done in large numbers, even though they may be individually inexpensive, account for a major part of the direct annual expenditure of most clinical laboratories. It is also clear from the Table that expenditure on major equipment, which on cursory inspection appears to be an expensive feature of modern laboratories, accounts for a surprisingly small part of test costs.

The conclusion is that the revenue consequences of purchasing the ISE analyser would be considerable, and much more important economically than the capital outlay (less than $£ 600$ per annum, assuming a 10-year lifespan). This is largely due to the cost of reagents which can only be obtained from the instrument manufacturer. Furthermore, because of the high cost of the urine diluent $(£ 0.67$ per test in this example), the extent of the revenue consequences will depend to a large extent on the demand for urinary as opposed to serum assays. These drawbacks to the ISE analyser, which may well be attractive otherwise because of its speed of operation, become apparent only after consideration of the detailed costing figures. Note that the most appropriate figure for the laboratory to use in deciding between alternative analytical methods or equipment is the total annual expenditure on this type of test.

By contrast, the figure needed by the clinician who wishes to know the costs generated by a particular type of request is the total cost per request: in this example, $£ 5.23$ for flame photometry and $£ 5.32$ with the ISE analyser. The small proportional difference is a simple consequence of the fact that overhead or indirect costs comprise the major part of most laboratory costs, so that it would be grossly misleading to ignore them in any consideration of the cost of a determination.

\section{EFFECT OF WORKLOAD ON COSTS}

When predicting the additional expenditure needed to meet increases in workload, many managers intuitively assume an approximately linear relationship. At the same time, efficiency is widely believed to improve as equipment and staff are used more fully, since this reduces the cost per test. Unfortunately, however, demand may be stimulated by this apparent reduced cost and the total expenditure thereby further increased. It is therefore important to understand the relation between costs, expenditure and workload.

Data given elsewhere ${ }^{1}$ and in Tables 5 and 7 indicate that the direct cost per test, of both labour and consumables, decreases as workload increases in a manner which depends on the type of test. Expenditure on consumables can often be confidently predicted by listing them in detail as in Table 6, but for some tests this is more difficult. With assay kits, for example, the amount of reagent prepared should ideally match the daily workload. This means that the analysis of the $21 \mathrm{st}$ specimen, when the reagent vial is only sufficient for 20 , will produce a stepwise increase in expenditure and cost per test. On the other hand, assay of only 10 specimens will result in wastage and also increase the cost per test. In practice, the daily workload varies and the average must be calculated over a representative period.

So long as a test remains in the laboratory repertoire, reagents must be purchased and staff be available to do it. A supposedly effective method of achieving savings is to delete unwanted or uninformative tests from the repertoire altogether, but unless the number of staff is reduced, there will be no net saving in labour costs because the direct labour saved will either be added to the reservoir of indirect labour or deployed on a different test. As a result, costs will be redistributed but expenditure will not change. Savings in expenditure would then be less than anticipated from a simplistic prediction 
Table 7 Direct costs (£) per test in laboratories $A(1980 / 81), B$ (cf Table 4) and C (1979/80) expressed at 1981/82 prices

\begin{tabular}{|c|c|c|c|c|c|c|c|c|c|c|}
\hline \multirow[t]{2}{*}{ Test } & \multirow[t]{2}{*}{$n$} & \multicolumn{4}{|c|}{ Direct cost per test } & \multirow[t]{2}{*}{$n$} & \multicolumn{4}{|c|}{ Direct cost per test } \\
\hline & & Con & $L a b$ & Cap & Total & & Con & $L a b$ & Cap & Total \\
\hline & & \multicolumn{4}{|c|}{ Laboratory $A$} & & \multicolumn{4}{|c|}{ Laboratory $B$} \\
\hline Blood alcohol & 182 & 0 & $2 \cdot 18$ & 0 & $2 \cdot 18$ & 53 & 7.02 & $4 \cdot 26$ & 0 & $11 \cdot 28$ \\
\hline Faecal fat & 104 & 0.98 & $2 \cdot 14$ & 0.91 & $4 \cdot 03$ & 59 & 0.57 & 2.93 & $2 \cdot 03$ & $5 \cdot 53$ \\
\hline Acid phosphatase & 924 & 0.06 & 0.57 & 0 & 0.63 & 389 & 0 & $1 \cdot 60$ & 0 & 1.60 \\
\hline Magnesium & 78 & 0.84 & 1.08 & 0 & 1.92 & 421 & 0 & 0.43 & $4 \cdot 40$ & $4 \cdot 83$ \\
\hline Amylase & 468 & 0.32 & $1 \cdot 13$ & 0 & 1.45 & 443 & 0 & 0.62 & 0 & 0.62 \\
\hline Calcium (urgent) & 52 & 0.02 & $2 \cdot 02$ & $0 \cdot 80$ & $2 \cdot 84$ & 1156 & 0 & $1 \cdot 08$ & 0 & $1 \cdot 08$ \\
\hline Lithium & 768 & 0 & 0.46 & 0 & 0.46 & 1600 & 0 & 1.09 & 0.07 & $1 \cdot 16$ \\
\hline Phosphate & 52 & $\begin{array}{l}0.02 \\
L a b o\end{array}$ & $\begin{array}{ll}1 \cdot 08 \\
-1.9\end{array}$ & 0.81 & $1 \cdot 91$ & 4435 & $\begin{array}{l}0 \\
\text { Labo }\end{array}$ & 0.76 & $0 \cdot 36$ & $1 \cdot 12$ \\
\hline Lactate dehydrogenase & 2002 & $0 \cdot 14$ & \multicolumn{3}{|c|}{ Laboratory $A$} & 854 & \multicolumn{3}{|c|}{ Laboratory $C$} & $1 \cdot 29$ \\
\hline Creatine kinase & 104 & $1 \cdot 27$ & 0.86 & 0.28 & $2 \cdot 41$ & 1294 & 1.44 & 0.79 & 0.28 & $2 \cdot 51$ \\
\hline Cholesterol & 1618 & 0.60 & 0.39 & 0.28 & $1 \cdot 27$ & 4800 & 0.26 & $0 \cdot 26$ & 0.09 & 0.61 \\
\hline Triglycerides & 1618 & 0.39 & $0 \cdot 38$ & 0.29 & $1 \cdot 06$ & 5324 & $0 \cdot 58$ & 0.44 & $0 \cdot 28$ & $1 \cdot 30$ \\
\hline Profile $\left\{\begin{array}{l}10 \text { channel } \\
11 \text { channel }\end{array}\right.$ & 28990 & 0.56 & $0 \cdot 18$ & 0.41 & $1 \cdot 15$ & 51119 & 0.58 & $0 \cdot 28$ & 0.30 & $1 \cdot 16$ \\
\hline
\end{tabular}

$\mathrm{n}=$ total number of tests or test groups performed in the year.

from the total cost of the test deleted.

Rarely performed tests necessarily account for a small proportion of the annual expenditure, even if they are "expensive" on a cost per test basis (Table 4). For example, deletion of blood ammonia assay (with a direct cost per test of $£ 12.01$ ) from the repertoire of laboratory $B$ would result in negligible savings because the cost of consumables is virtually zero. On the other hand, deletion of thyroxinebinding globulin assay (with a direct cost of only $£ 1.78$ per test), relatively frequently performed in laboratory B, would immediately save $3748 \times £ 1.13$ $=£ 4235$ per annum in consumables. Thus, immediate savings can be achieved most effectively through those tests which entail a high annual expenditure on consumables, and it is these tests on which attention should be focused in attempts either to reduce demand or to find a source of cheaper reagents.

\section{EFFECT OF LABORATORY ORGANISATION ON COSTS}

Changes in laboratory organisation and practice have a profound effect on costs which, from the laboratory's standpoint, can often be assessed by simple arithmetic and taking into account all apparent consequences of the change. However, cost analysis of laboratory tests without consideration of their clinical use and the speed with which they are reported will give a distorted picture of overall costs to the hospital or the health service as a whole. ${ }^{6}$

Most tests are done in batches, and the unit cost is smaller for large batches than for single analyses. As a result, some laboratories tend to accumulate specimens for non-urgent tests and run them in a single batch at say weekly intervals, or send them away to a larger centre. Although this will save money for the laboratory, it may incur costly clinical delays, particularly if the patient is kept in hospital to await the result at a current cost of about $£ 80$ per day.

Similar considerations apply in assessing the extra cost of tests performed out of hours. With the present system of payment used in the UK for out-ofhours work, these costs, which are mainly for labour, are directly related to workload, and immediate cask savings could be made by reducing the number of out-of-hours tests-but only if this had no adverscost consequences in the clinical sphere.

Investigation of patients before admission to hospital, and the rapid provision of assays such as serum drug levels for outpatients, can often be made at little or no extra expense for the laboratory, but may save clinical costs. The complexity of these nonlaboratory costs, and the difficulty of measuring them, constitute an unmet challenge in making a complete assessment of the overall cost consequences of performing a test at once or after a delay.

Similar considerations arise in assessing the cost/ benefit of performing a fixed battery of tests whenever any one of them is requested. The cost of a biochemical profile is remarkably small when performed on a modern multichannel automated analyser (Table 7), and indirect costs are reduced because of the simplification of specimen separation, work organisation and reporting. However, the present popularity of profiling amongst laboratory workers overlooks two important factors.

First, although the use of multichannel analysers reduces the apparent cost of each test, this is of no value if the test results are irrelevant or even misleading for individual patients. There is at present no evidence that the faster turnround of tests achieved by profiling produces any significant clinical savings. Moreover, any additional investigation 
induced by unrequested, unexpected results will entail additional expenditure, often without clinical benefit. On the other hand, to do such tests only when requested may result in delays, and will increase the indirect costs of specimen separation, sorting, etc. Consequently the full cost implications of including or excluding an analyte in a profile are far from straightforward. Equipment for automated discretionary testing is now becoming available, and it will be important to assess how this will affect laboratory and clinical costs.

A second consequence of the use of multichannel analysers is that, although each test or profile is inexpensive, profiles are done in large numbers, so that the annual expenditure on them is large. This perhaps provides justification for the widely held belief that automatic analysers are expensive whereas, on a cost per test basis, they are not. The availability of such equipment, able to do large numbers of cheap tests, stimulates demand, so that the total expenditure on them rises until it consumes a major part of the laboratory's budget.

In assessing the cost-effectiveness of laboratories, and the effect of changes in practice and organisation, it may be useful to compare data from different laboratories (Table 3). To the administrator, using the only data available to him, the comparison figure would be the total revenue expenditure divided by the number of requests - that is, the average revenue cost per request: $£ 3.99$ for laboratory $A$ and $£ 6.31$ for laboratory $\mathrm{B}$. The $60 \%$ difference is explainable on the grounds that laboratory $\mathrm{B}$ is an independent clinical chemistry department, with medical staff, in a teaching hospital, whereas laboratory $\mathrm{A}$ is located in a multidisciplinary department in a District General Hospital and has no medical staff. However, this explanation takes no account of differences in actual investigations performed in the two laboratories, and a more detailed analysis is more revealing.

The average direct cost per request (a measure not available without detailed cost analysis) in the two laboratories again differs, though in lesser degree (43\%): $£ 1.40$ for $A$ and $£ 2.01$ for B. However, greater differences emerge when the costs of individual tests are compared (Table 7). For many analytes (blood alcohol, faecal fat, acid phosphatase, calcium), the cost per test is, predictably, lower in the laboratory where it is done in larger numbers, but there are additional marked differences between $A$ and $B$ which are probably method-related. Although a few generalisations about the cost per test can be made from these data, the figures could by no stretch of the imagination be used as a basis for a joint, let alone a national, price list.

There are interesting differences between
Table 8 Annual expenditure ( $f$ ) on consumables for $L D$ and $C K$ assays in laboratories $A(1980 / 81)$ and $C(1979 / 80)$, expressed at $1981 / 82$ prices

\begin{tabular}{|c|c|c|}
\hline & $\begin{array}{l}\text { Laboratory } \\
\text { A }\end{array}$ & $\underset{C}{\text { Laboratory }}$ \\
\hline \multicolumn{3}{|l|}{ Lactate dehydrogenase } \\
\hline $\begin{array}{l}2002 \text { tests pa at } £ 0.14 \text { each } \\
\text { Creatine kinase }\end{array}$ & 280 & - \\
\hline 1294 tests at $f 1.44$ each & - & 1863 \\
\hline 104 tests at $£ 1.27$ each & 132 & - \\
\hline Total expenditure per annum & 412 & 2230 \\
\hline
\end{tabular}

laboratories $\mathrm{A}$ and $\mathrm{C}$ in the cost and workload of serum lactate dehydrogenase (LD) and creatine kinase (CK) assays (Table 8). The annual expenditure on consumables for the two enzymes together is considerably greater in laboratory $\mathrm{C}$, although the total number of enzyme assays $(2106,2148)$ is similar. This is largely due to the greater demand for CK (with a higher direct cost per test) in laboratory C. Since these two tests are mainly used in the diagnosis of myocardial infarction, it would be interesting to know the reasons for this difference in clinical request pattern, and whether it was reflected in clinical benefit. If the clinical staff using laboratory $\mathrm{C}$ were to adopt the request pattern used in laboratory $\mathrm{A}$-that is, $\mathrm{LD}$ in preference to $\mathrm{CK}$ - the annual expenditure on consumables in laboratory $\mathrm{C}$ would be considerably reduced.

\section{Laboratory costs and the clinician}

Few clinicians know the cost of the tests they request for their patients. The main reason for this is that laboratory directors themselves do not have this information, and when expenditure rises tend to blame the clinician for imposing additional demands without realising their cost implications. Often laboratories contribute to this polarisation by a simplistic cost analysis which makes tests appear cheaper than they are.

What answer should the head of a laboratory give to the clinician or medical student who asks "What is the cost of Test X?" The question is deceptively simple, but several equally valid answers are possible. In the first place, it will be clear from the foregoing that the answer will be different for each laboratory, and that a guess which ignores the less obvious indirect costs will be misleading.

The simplest general answer is to state, for that laboratory, the average total cost per request for that test, ie the direct costs of consumables, labour and capital, plus the "handling charge" for indirect costs. Thus for laboratory B (Table 4) the average cost of a request for serum digoxin assay is $£ 10.24$ (total direct) plus $£ 4.80$ (indirect), making a total of 
£14.04. Although the figure will be higher for an out-of-hours request, the average figure serves as a general guide in answering the clinician's question. It represents the without-profit "marketplace" cost, which should not be too dissimilar from the price charged by commercial laboratories. The element of profit built into the commercial price is approximately matched by the cost of the research, advisory and consultative functions provided by the hospital laboratory.

The main reason for higher laboratory costs over the past few years is the increased number of requests made by clinicians. ${ }^{578}$ Attempts to modify clinicians' requesting patterns have not met with great success ${ }^{9-11}$ and whether clinicians would be greatly influenced by knowing the cost of a test-for example, if this were printed on the laboratory report-is at the least dubious. ${ }^{11-13}$ Tests which appear relatively cheap on a unit basis may easily lead to additional demands, and if these demands are readily met with high-throughput automated equipment, total expenditure will increase. For this reason the total cost for a particular request is the most appropriate figure to give to the interested clinician, not the "direct cost per test" which is often demanded by planners and quoted by manufacturers of equipment and reagents.

Estimates of the potential savings which would result from discontinuing unnecessary investigations $\mathrm{s}^{14}{ }^{15}$ are often equated with the average cost per request (which is the only figure available). This is highly misleading since in most cases the only real saving in the short term in discontinuing a test is in direct consumable costs, which are usually a small proportion of total costs: that is, marginal costs are equal to marginal savings.

On the other hand, the cost of setting up a new assay which is not in the laboratory's repertoire is much greater, since additional equipment may be needed or extra staff employed.

Knowledge of the costs of alternative diagnostic strategies is of direct interest and importance to the clinician. For example, one hospital may use lactate dehydrogenase assays, which are relatively cheap, as the main enzyme test in the diagnosis of myocardial infarction, whereas another may use creatine kinase, which is more expensive. Although the difference in the cost per individual test appears small, the difference in laboratory expenditure becomes highly significant when large numbers of tests are done annually.

\section{Conclusions}

At present, the concept of laboratory costs has different meanings to different people. The worker at the bench will naturally be conscious only of the cost of what he is doing, and will be oblivious to most indirect costs, which account for most of the expenditure. The laboratory manager is concerned with budget. Costing data can help in showing how money is spent, in assessing alternative methods and work patterns, and pinpointing where savings might be made. The hospital or district administrator is concerned with the total cost of providing a pathology service and with balancing this against the needs of other departments. Often the only person who perceives all these costs, and can estimate their relative importance, is the laboratory director, who is usually untrained in economics or accountancy.

In assessing how useful costing data can be, it is essential to define questions which have sizeable financial implications. For example, marginal costs (except for out-of-hours work) are usually small and the information they yield is rarely helpful, particularly when the clinical savings which might result from doing one extra test are left out of account.

The costing method of Broughton and Hogan yields several different costing quotients and totals. Each of these has its uses. The total cost per request represents the marketplace cost of completely fulfilling that request. It is the most appropriate figure to present to clinicians and administrators and for use in billing. The average total revenue cost per request is the simplest of all figures to calculate andō for many large-scale administrative purposes-for? example, specialty costing-may be perfectly adequate. The direct revenue cost per test is of limited value; more useful is the annual direct revenue expenditure for each type of test, which reflects the laboratory resources devoted annually to that type of test. This is important in assessing the revenue consequences of changing workloads, analytical methods or equipment. The direct consumable cost per test is relevant in assessing marginal costs, but the annual direct consumables expenditure on each type of test is what is needed in deciding where immediate savings can be sought.

Although the method ${ }^{1}$ outlined here has so far been applied only to clinical chemistry laboratories, it should be applicable to other laboratory disciplines and hospital service departments. Many of these have the same categories of direct and indirect expenditure, and face similar questions of cost as those described here. Clearly, there would be major advantages if all agreed to use the same approach and terminology.

The authors thank the many friends and colleagues who have contributed with ideas or data on costs and costing. 
Financial support of the Wolfson Research Laboratories by the Department of Health and Social Security is gratefully acknowledged.

\section{References}

${ }^{1}$ Broughton PMG, Hogan TC. A new approach to the costing of clinical laboratory tests. Ann Clin Biochem 1981;18:330-42.

${ }^{2}$ Department of Health and Social Security. Procedure for determining test costs in pathology laboratories (Cooper-Lybrand report). London: DHSS, 1975.

${ }^{3}$ Stilwell JA. Costs of a clinical chemistry laboratory. J Clin Pathol 1981;34:589-94.

4 Krieg AF, Israel M, Fink R, Shearer LK. An approach to cost analysis of clinical laboratory services. Am J Clin Pathol 1978;69:525-36.

${ }^{5}$ Griner PF, Glaser RJ. Misuse of laboratory tests and diagnostic procedures. N Engl J Med 1982;307:1336-9.

${ }^{6} \mathrm{Klatt}$ EC, Wasef ES, Wong ET. Creatine kinase in a biochemical test panel. The high cost of a seemingly inexpensive test. Am J Clin Pathol 1982;17:280-4.

' Hardwick DF, Morrison JI, Tydeman J, Cassidy PA, Chase WH. Laboratory costs and utilisation: a framework for analysis and policy design. J Med Educ 1981;56:307-15.
' Fleming PR, Zilva JF. Workloads in chemical pathology: too many tests? Health Trends 1981:13:46-9.

${ }^{9}$ Eisenberg JM, Williams SV. Cost containment and changing physicians' practice behavior. JAMA 1981;246:2195-2201.

${ }^{10}$ Martin AR, Wolf MA, Thibodeau LA, Dzau V, Braunwald E. A trial of two strategies to modify the test-ordering behavior of medical residents. $N$ Engl J Med 1980;303:1330-6.

" Grivell AR, Forgie HJ, Fraser CG, Berry MN. Effect of feedback to clinical staff of information on clinical biochemistry requesting patterns. Clin Chem 1981;27:1717-20.

${ }^{12}$ Forest JB, Ritchie WP, Hudson M, Harlan JF. Cost containment through cost awareness: a strategy that failed. Surgery ( $S t$ Louis) 1981;90:154-8.

${ }^{13}$ Cohen DI, Jones P, Littenberg B, Neuhauser D. Does cost information availability reduce physician test usage? Med Care 1982;20:286-92.

${ }^{14}$ Sandler G. Costs of unnecessary tests. Br Med J 1979;ii:21-4.

is Young DW. An aid to reducing unnecessary investigations. $B r$ Med J 1980;i:890-2.

Requests for reprints to: Mr PMG Broughton, Wolfson Research Laboratories, Queen Elizabeth Medical Centre, Edgbaston, Birmingham B15 2TH, Englar,d. 